\title{
Neuartiges Konzept zur Schweißparameterregelung beim Widerstandspunktschweißen
}

\author{
Nowoczesna koncepcja regulacji \\ parametrów zgrzewania oporowego punktowego
}

\section{Streszczenie}

Przeprowadzono analizę wpływu szczeliny między łączonymi blachami ze stali o różnej wytrzymałości w procesie punktowego zgrzewania konwencjonalnego i zgrzewania z zastosowaniem sterowania geometrią zgrzeiny i strefy wpływu ciepła na jakość złączy. Wykazano, że nowy proces zgrzewania punktowego z systemem regulacji geometrii zgrzeiny i strefy wpływu ciepła jest szczególnie korzystny w przypadku łączenia stali o wysokiej wytrzymałości, zwłaszcza przy większej grubości blach.
Abstract

The analysis of influence of the gap between the joining steel plates of varying strength in the conventional spot welding and spot welding with weld and heat-affected zone geometry control on the welds quality is presented. It is shown that the new spot welding process with the weld and heat affected zone geometry control system is particularly advantageous for high strength steel joining, especially for thick plates.

\section{Einleitung}

Das Widerstandspunktschweißen ist ein weit verbreitetes Fügeverfahren in der Automobilindustrie beim Karosseriebau aus Stahl. Durch die hohe Wirtschaf-tlichkeit des Prozesses auf Grund eines hohen Automatisierungsgrades sowie das Fehlen von Schweißzusätzen und Schutzgasen hat dieses Schweißverfahren eine bedeutende Stellung erlangt. Im Weiteren entsteht beim konventionellen Schweißprozess eine geringe Menge an gesundheitsschädlichen Gasen

Dipl. Ing. Pawel Baryliszyn, Dipl. Ing. Thomas Noack - Volkswagen Aktiengesellschaft, Niemcy, mgr inż. Krzysztof Janus - Volkswagen Poznań, prof. dr hab. inż. Jerzy Nowacki - Zachodniopomorski Uniwersytet Technologiczny w Szczecinie. daher ist keine hocheffizientes Ventilationssystem notwendig. Weiterhin verursacht der Schweißvorgang nur eine geringe Lichtemission. Die Anzahl der Widerstandsschweißpunkte an einer modernen Rohkarosse beträgt zwischen 3000 und 5000 [1].

Die Tabelle 1 stellt den Anteil von Fügeverfahren an einer modernen Fahrzeugkarosserie am Beispiel des Audi A4 dar. An 3. Stelle steht hier auf Grund hervorragender Eigenschaften in der Dünnblechverarbeitung das Laserschweißen. Dieses Verfahren verdankt seinen Aufschwung der Anwendung in der Automobilindustrie. Mittels des Laserstrahles werden die Bauteile ausschließlich lokal erwärmt und dadurch bei einer relativ hohen Prozessgeschwindigkeit quasi verzugsarm verschweißt. Eine große Herausforderung stellt das Schweißen von verzinkten Materialen dar, da das Verdampfen des Zinkbelages die Entstehung von Schweißspritzern und -poren verursacht. Zur Gewährleistung einer gleichbleibenden Qualität muss beim Laserschweißen mit Hilfe von aufwendiger Spanntechnik 
Tabelle I. Verteilung verschiedener Fügeverfahren im Karosseriebau am Beispiel des Audi A4 [2]

\begin{tabular}{|l|c|}
\hline Widerstandspunktschweißen & $\begin{array}{l}5000 \text { Pkt. }(\approx 225 \mathrm{~m} \text { bei } \\
\text { Punktabstand } 45 \mathrm{~mm})\end{array}$ \\
\hline Strukturkleben & $90 \mathrm{~m}$ \\
\hline Laserstrahlschweißen & $4 \mathrm{~m}$ \\
\hline Plasmalöten & $3,8 \mathrm{~m}$ \\
\hline MAG - Schweißen & $0,77 \mathrm{~m}$ \\
\hline Laserlöten & $0,65 \mathrm{~mm}$ \\
\hline
\end{tabular}

ein Blechabstand von genau 0,1 bis 0,2 mm zwischen den zu fügenden Bauteilen zur Entgasung eingestellt werden, wodurch die Prozesskosten steigen [3].

Im Gegensatz dazu wird beim Widerstandspunktschweißen das flüssige Zink aus der Fügezone sowie Kontaktflachen des Werkstücks mit den Elektrodenkappen durch die Anpresskraft beider Elektroden nach außen verdrängt, wodurch ein dichter Mantel zum Schutz vor der Oxidation herum entsteht. In Verbindung mit der Abkühlphase während der Nachhaltezeit wird die Porenentstehung verhindert.

\section{Problemstellung}

Die erwähnte Problematik des negativen Einflusses vom Spalt zwischen den zu fügenden Blechen betrifft ebenso das Widerstandspunktschweißen. Größere Passungsprobleme der Bauteile führen zur Veränderung des elektrischen Widerstandes über die effektive Anpresskraft in der Fügeebene. Im Weiteren werden die Bauteile mit Maßabweichungen im Laufe des Schweißprozesses verzogen und unter Spannung verschweißt. Die Spaltentstehung nimmt mit der Bauteilkomplexität und Werkstofffestigkeit zu, Abbildung 1. Bei größeren Spalten kann eine prozesssichere Spaltüberbrückung mit dem konventionellen Schweißverfahren nicht hergestellt werden. Der Lösungsansatz ist eine veränderte Prozessführung, welche einen normgerechten Schweißpunkt unter Berücksichtigung der vorliegenden Passungssituation durch die Anpassung von Schweißparameter sicherstellt.

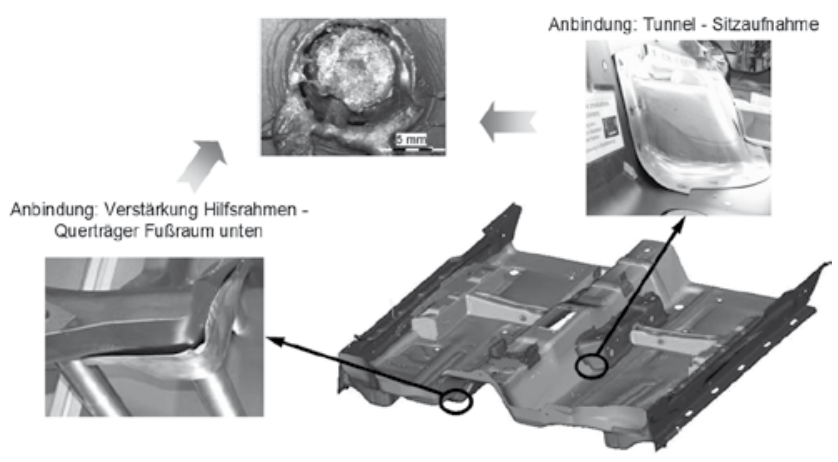

Abb. 1. Spaltentstehung beim Fügen komplexer Bauteile mit Toleranzen [4]
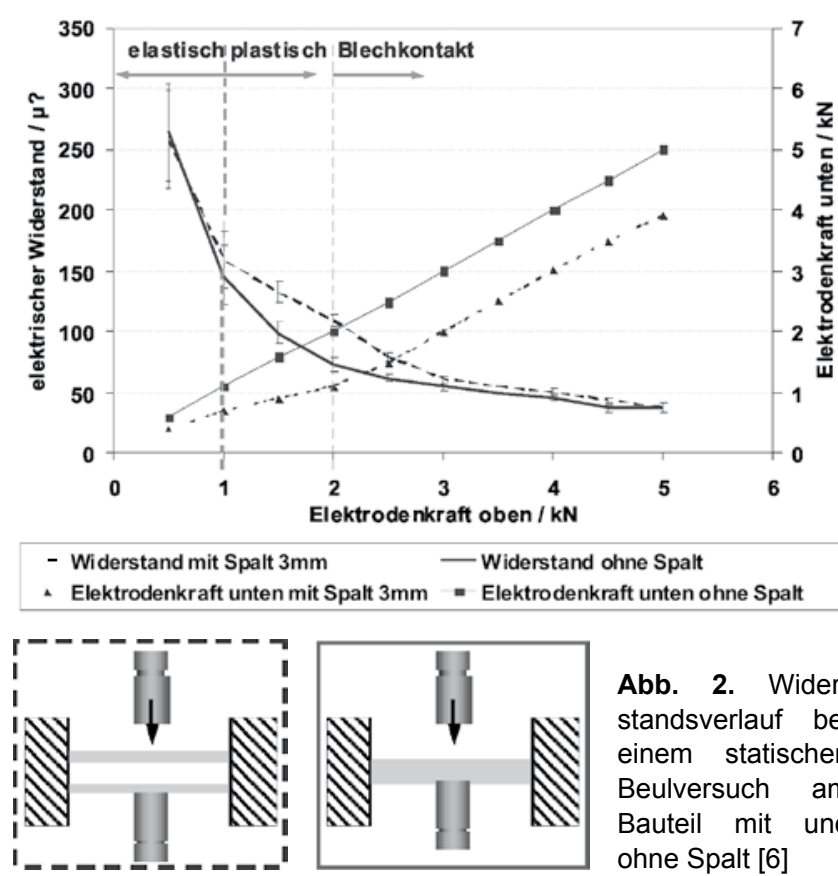

Abb. 2. Widerstandsverlauf bei einem statischen Beulversuch am Bauteil mit und ohne Spalt [6]

Der quasistatische Druckversuch in [6] beweist einen Zusammenhang zwischen dem gemessenen Übergangswiderstand und der Festigkeitsgrenze des Werkstoffes bei Toleranzproblemen. In der Abbildung 2 ist bei $3 \mathrm{~mm}$ Spalt am Beispiel des HX340LAD+Z100 eindeutig der Übergang von der elastischen in die plastische Beulung zu erkennen. Die Änderung des elektrischen Widerstandes entsteht durch das Eindringen der Elektrodenkappe in den Grundwerkstoff und demzufolge der Zunahme der Kontaktfläche von Elektrodenkappen.

\section{Anforderungen aus dem Karosseriebau}

Die Reaktionen des Grundwerkstoffes auf den Schweißprozess zusammen mit der konstruktiven Gestaltung von Schweißverbindungen definieren die Anforderungen an den Schweißprozess. Moderne Werkstoffe im Karosseriebau müssen hohe Ansprüche nicht nur im Bezug auf die Beschaffungskosten, eine kostengünstige Verarbeitung zu Bauteilen, sondern auch die Festigkeit erfüllen. Mit ca. $40 \%$ des Gesamtgewichts stellt eine zeitgemäße Rohkarosserie das größte Potential zur Gewichtsreduzierung für zukünftige Produktinnovationen dar. Eine Reduzierung dieses Anteils führt zur Verbesserung der Fahrdynamik und Verringerung des Fahrzeugverbrauchs. Dabei darf die Sicherheit von Insassen nicht beeinträchtigt werden [1].

Die Fahrzeughersteller verwenden im Produktionsprozess verschiedene Werkstoffe von Stahl, über Leichtmetalllegierungen (z.B. Aluminium-, Magnesiumlegierung) bis hin zu Kunststoffen oder Verbundwerkstoffen (z.B. Kohlenstofffasern). Obwohl alle Materialien gewisse Vor- und Nachteile haben, wird der Stahl am

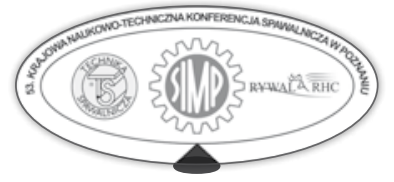


häufigsten in der Automobilindustrie eingesetzt, weil er ein relativ preiswerter Werkstoff und einfach in der Verarbeitung ist. Weiterhin können beim Material alle bewährten Verbindungstechnologien mit dem hohen Reifegrad zum Einsatz kommen. Abhängig von der verwendeten Stahlsorte wird ein breiter Festigkeitsbereich bis zu Rm = 1650 MPa gedeckt [7, 8]. Dies ermöglicht eine optimale Anpassung von Werkstoffeigenschaften an die Anforderungen des jeweiligen Karosseriebauteils. Die Abbildung 3 stellt für ausgewählte Stahlsorten mechanische Werkstoffeigenschaften dar. Die Anwendung alternativer Werkstoffe erfordert dagegen den Einsatz von neuen Verbindungsverfahren.

In den ersten Automobilen war für die Kunden die Insassensicherheit unwichtig, da die damals erreichten Höchstgeschwindigkeiten deutlich geringer als heutzutage waren. Ein großer Wert wurde auf den Komfort, die Dynamik und natürlich Kosten gelegt. Die nicht selbstragende Fahrzeugkarosserie wurde bei diesen Fahrzeugen meistens auf einem steifen Rahmen aus

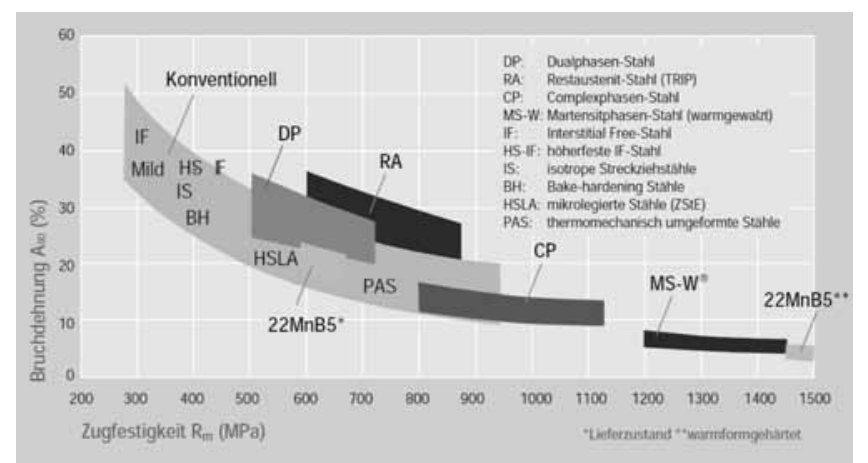

Abb. 3. Leistungsspektrum kalt- und warmgewalzter Stahlsorten in der Automobilindustrie [9]

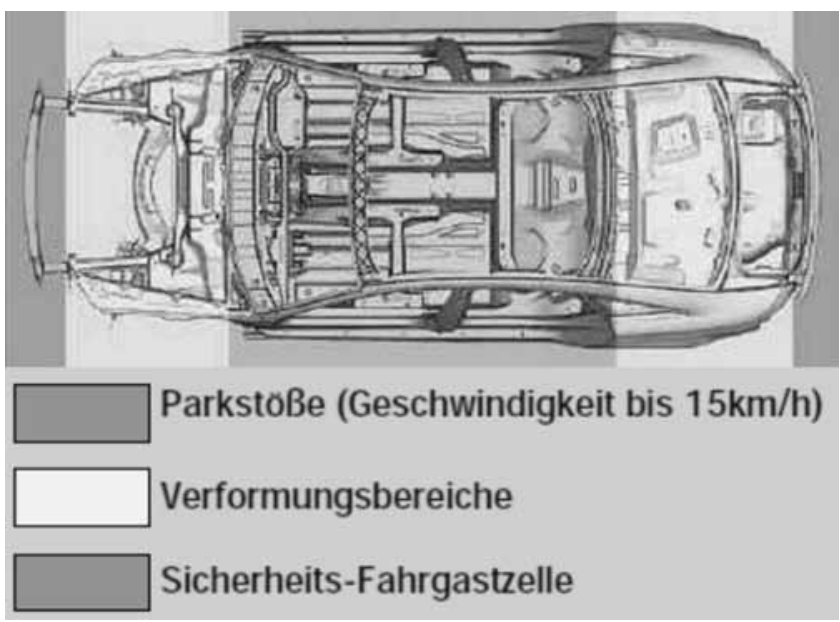

Abb. 4. Passive Sicherheitsbereiche eine Fahrzeugkarosserie [10]

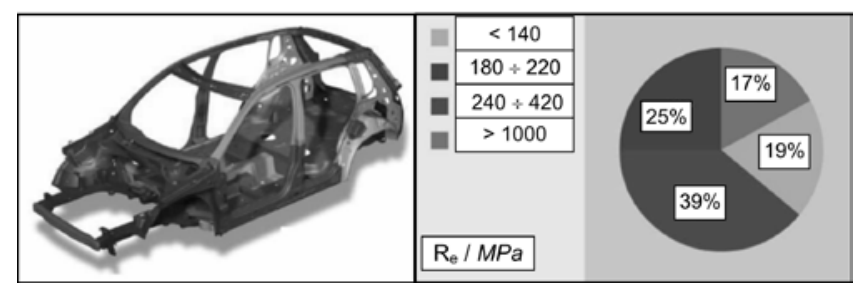

Abb. 5. Anwendung unterschiedlicher Werkstofffestigkeiten in der Struktur einer moderner Karosserie am Beispiel VW Tiguan [1]
Stahlprofilen aufgebaut. Massive Stoßfänger dienten lediglich dem Schutz bei leichten Kollisionen. Eine moderne, selbstragende Fahrzeugkarosserie besteht aus mehreren passiven Sicherheitsbereichen, die verschiedene Funktionen bei einer Kollision erfüllen (Abbildung 4). Beispielweise werden in den Deformationszonen Stahlsorten mit einer Festigkeit bis $420 \mathrm{MPa}$ und in der Sicherheits-Fahrgastzelle über $1000 \mathrm{MPa}$ eingesetzt, welche den enormen Kräften beim Unfall standhalten können [1].

Die blau markierte Zone schützt vor leichten Stößen z.B. beim Parken, welche bei einer Geschwindigkeit unter $15 \mathrm{~km} / \mathrm{h}$ geschehen. Gelb dargestellt ist die tatsächliche Deformationszone. Durch eine plastische Verformung bei einer Kollision wird die kinetische Aufprallenergie absorbiert und verteilt. Die Sicherheits- Fahrgastzelle (grün) ist dagegen eine sehr feste und steife Konstruktion aus dem Tunnel, Schwellern sowie Säulen. Durch den Einsatz verschiedener Blechgüten wird die erforderliche Bauteilsteifigkeit erreicht, ohne dabei die Blechstärke zu vergrößern. Mit diesem Ansatz können stetig steigende Anforderungen an die Sicherheit bei gleichbleibendem oder sogar reduziertem Fahrzeuggewicht erfüllt werden. Die Abbildung 5 zeigt die Verteilung verschiedener Stahlgüten in der Struktur einer modernen PKW-Karosserie am Beispiel des VW Tiguan.

Mit Hilfe der Fügetechnik müssen sehr komplexe Konstruktionen heutiger Fahrzeugkarosserien bestehend aus vielen Einzelteilen und verschiedenen Stahlsorten mit unterschiedlichen Eigenschaften miteinander prozesssicher verbunden werden. Um eine normgerechte Schweißverbindung nach [11, 12, 14, 15] herzustellen, ist eine genau Bauteilpassung besonders bei einer höherer Werkstofffestigkeit von Vorteil. Dies solIte durch konstruktive Maßnahmen z.B. Materialkombinationen, Fügefolgen oder Schweißpunkposition in der Planungsphase ermöglicht werden. Hierbei sollten Schweißpunkte an Bauteilkanten und in jeglichen Rundungen vermieden werden. Liegt zwischen den zu fügenden Bauteilen ein Spalt während des Widerstandspunktschweißens vor, geht ein gewisser Teil der eingestellten Anpresskraft für die elastische Bauteildeformation verloren und steht dem Prozess nicht mehr zur Verfügung. Die effektive Anpresskraft ist umso geringer, je größer der Spalt zwischen den Bauteilen und je dicker und steifer diese selbst sind. Ein zu großer Abstand zwischen den Bauteilen führt zu fehlerhaften Schweißpunkten. Nach [13] liegt die zulässige Spaltgröße bei $0,8 \mathrm{~mm}$ bei Langzeitschweißungen und bei $0,3 \mathrm{~mm}$ bei Kurzzeitschweißungen.

Im Einzelfall wird in Anlehnung an [13] das Bauteilausrichten zur Spaltminimierung insbesondere bei Blechstärken von mehr $2 \mathrm{~mm}$ empfohlen. Es ist jedoch nur im Falle von Bauteilen aus Werkstoffen mit einer geringen Festigkeit und Materialstärke, welche eine einfache Geometrieform besitzen, möglich. Des Weiteren kann dieser Lösungsansatz nicht in der Massenproduktion angewendet werden, da die Taktzeit erheblich steigen würde. 


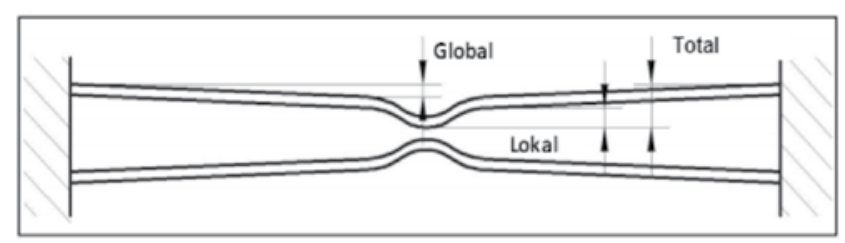

Abb. 6. Verformungsanteile am Flansch mit einem Spalt

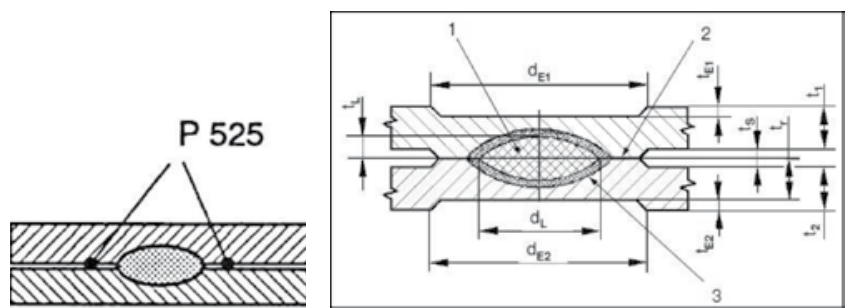

Abb. 7. Spaltdefinition nach ISO 6520-2 (links) und VW 01105-1 (rechts)

Wegen variablen Bauteiltoleranzen ist eine genaue Einstellung von Prozessparametern nur beding möglich und mit sehr hohen Kosten verbunden. Dies erfolgt entweder anhand von praktischen Versuchen an realen Bauteilen durch manuelle Prozessparameteranpassung oder während des Schweißvorganges mit Hilfe von adaptiven Regelkonzepten.

In der Abbildung 6 ist die entstandene Flanschverformung nach dem Widerstandspunktschweißen bei einem Spalt schematisch dargestellt. Die gesamte plastische Verformung der Bleche setzt sich aus der lokalen und globalen Verformung zusammen. Die lokale Deformation geschieht nach der Definition in $[5,6]$ ausschließlich im Bereich bis $8 \mathrm{~mm}$ von der Elektrodenkappenachse und die globale auf der restlichen Flanschfläche. Außer der plastischen tritt ebenfalls eine elastische Verformung des Schweißflansches auf. Dieser Anteil nimmt bei einer gleichbleibenden Elektrodenkraft mit der Streckgrenze des Stahls zu.

Fertige Schweißpunkte müssen entsprechende Qualitätsanforderungen erfüllen. Die Spaltproblematik ist in den gültigen Normen für das Widerstandsschweißen nicht genauer dargelegt. In den Normen $[14,15]$ wird versucht, mit dem Begriff „ein zu großer Spalt" diesen Prozessparameter zu definieren. Außer diesem Begriff fehlt in [14] jede Angabe über den zulässigen Maximalwert für die Störgröße dem Symbol P 525 (Abbildung 7). [15] legt genau den zulässigen Spalt $t_{S}$ (Abbildung 7 ) für eine Zweiblechverbindung nach dem Schweißvorgang anhand der verursachten Einschnürung der Wanddicke $t_{r}$ im Randbereich der Schweißlinse. Hierbei müssen auch die übrigen Anforderungen erfüllt sein.

\section{Zweistufiger Prozess}

Um den Einfluss des Spaltes am Schweißflansch auf den Schweißpunkdurchmesser beim herkömmlichen Widerstandsschweißprozess zu untersuchen, wurde eine Reihe von Schweißversuchen bei verschiedenen Spaltmaßen im Konstantstromregelmodus durchgeführt. Die Ergebnisse sind in der Abbildung 8 für HX340LAD +Z100MB und 22MnB5 +AS150 in 1,0 und $1,5 \mathrm{~mm}$ Materialstärke dargestellt. Erwartungsgemäß nimmt der Schweißlinsendurchmesser ohne Anpassung von Schweißparameter mit der zunehmenden Materialstärke und -festigkeit ab. Die horizontalen Linien markieren den Mindestpunktdurchmesser nach [15] untersuchter Blechstärken. Größte Schweißpunktdurchmesser wurden bei $1 \mathrm{~mm}$ Spalt erreicht, da durch eine leichte Blechverformung eine Steigerung der Stromdichte in der Fügezone entstand. Darüber hinaus wurde mehr Energie zur Schweißlinsenbildung in der gleichen Zeit eingebracht. Weitere Spaltvergrößerung verursacht wegen einer größeren Lokalblechverformung eine Verringerung des Punktdurchmessers bzw. der Verbindungsfestigkeit. Bei 5 $\mathrm{mm}$ Zwischenlagen nahmen die Kontaktflächen zu und der Mindestpunktdurchmesser wurde für alle Versuchswerkstoffe nicht mehr erreicht. Die Abbildung 9 zeigt die Bruchbilder beim Spalt von 0 und $5 \mathrm{~mm}$ für den HX340LAD +Z100 und 22MnB5 +AS150. Beim geringen Spalt kam es zum Versagen der Schweißverbindung durch das Ausknöpfen mit einem erheblichen Anteil des Grundmaterials, was auf eine ausreichende Festigkeit der Schweißverbindungen schließen lässt. Aus den Versuchen geht hervor, dass eine Änderung des Zwischenlageabstandes neben dem Schweißpunktdurchmesser auch das Verbindungsversagen beeinflusst. Dies war deutlich anhand von höher- und höchstfesten Werkstoffen zu erkennen. Beim höchstfesten Werkstoff in $1,5 \mathrm{~mm}$ Materialstärke verlief der herkömmliche Schweißprozess unstabil und es kam zu Löchern und Poren im Grundwerkstoff.

Die Tendenz zum Stoffleichtbau mit höchstfesten Stählen erfordert die Entwicklung neuartiger Anlagentechnik für die Automobilindustrie, um bei neuen Anforderungen die normgerechte Schweißpunktqualität in der Großserienfertigung weiterhin zu gewährleisten. Eine moderne Fahrzeugkarosserie besteht aus vielen komplexen Einzelteilen mit gewissen Fertigungstoleranzen. Dadurch können beim Fixieren in einer Spannvorrichtung an Schweißflanschen Spalte entstehen. Dieses Problem kann mit einer größeren Anpresskraft der Schweißelektroden gelöst werden, was jedoch mit Mehrkosten für leistungsfähigere Maschinen verbunden ist. Dabei muss aber übermäßige Deformation von Bauteilen in Kauf genommen werden. Weiterhin hat

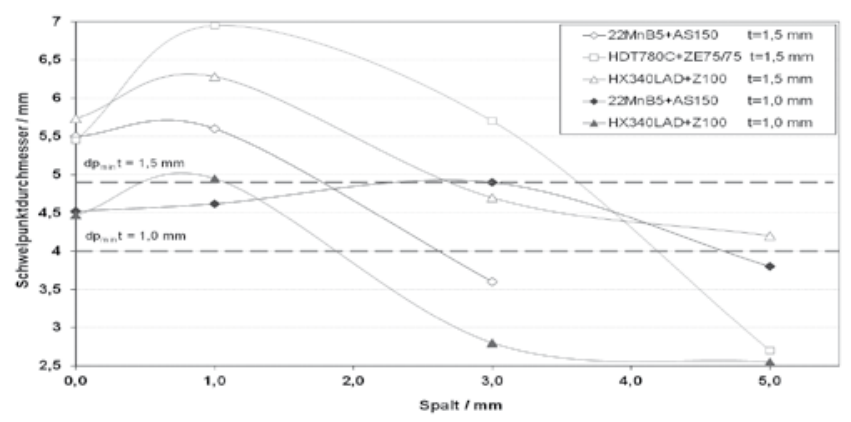

Abb. 8. Einfluss des Spaltes auf den Schweißpunktdurchmesser beim herkömmlichen Widerstandsschweißprozess

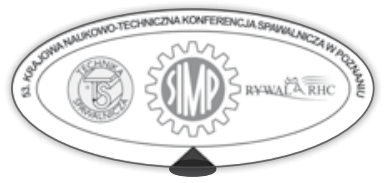


dieser Lösungsansatz die Entstehung von Spannungen an den Fügestellen zur Folge, was wiederum eine Rissbildung unterstützt. Eine Vergrößerung der Elektrodenkraft verringert den elektrischen Übergangswiderstand im Bereich der Fügezone, wodurch eine Anpassung elektrischer Schweißparameter erforderlich ist.

Im Rahmen dieses Projektes wurde eine veränderte, zweistufige Prozessführung beim Widerstandspunktschweißen von Bauteilen mit Maßabweichungen untersucht. Im ersten Prozessschritt wird der vorliegende Spalt bei einer geringen Elektrodenkraft und Stromstärke im Vergleich zum herkömmlichen Schweißvorgang durch eine überwiegend lokale Beule quasi spannungsfrei überbrückt. Die Dauer des Stromflusses ist dafür länger. So verformte Bleche können anschließend mit einer geringen Elektrodenkraft konta-ktiert und prozesssicher verschweißt werden. Das Prinzip des zweistufigen Prozesses und die erzeugte Flanschgeometrie nach beiden Prozessschritten sind in der Abbildung 10 dargestellt.

Der zeitliche Ablauf der neuartigen Prozessführung ist in der Abbildung 11 schematisch gezeigt. Der

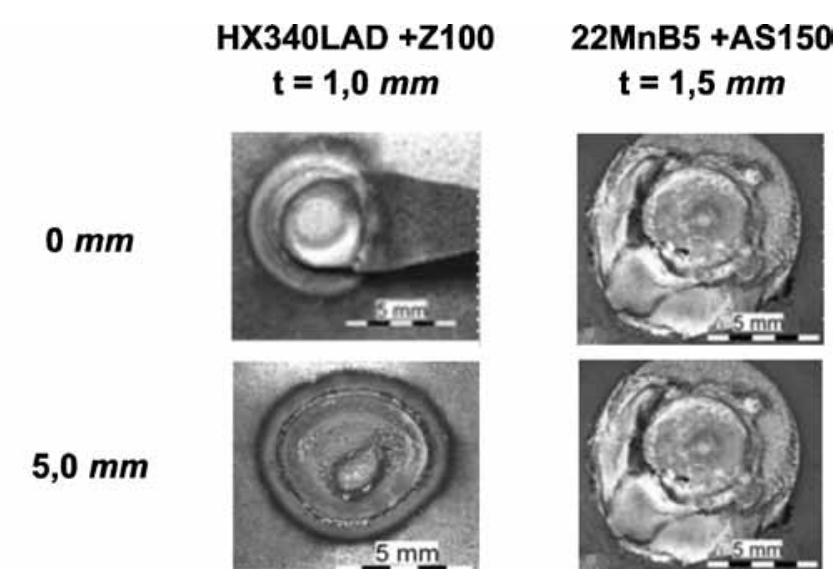

Abb. 9. Bruchbilder des HX340LAD +Z100 und 22MnB5 +AS150 bei 0 und $5 \mathrm{~mm}$ Spalt

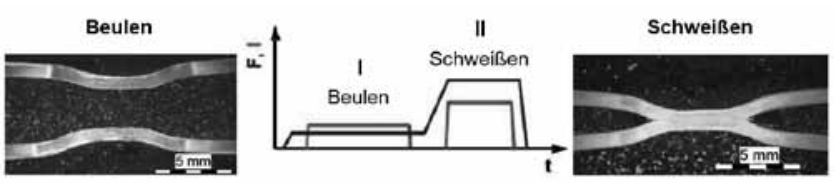

Abb. 10. Schematische Darstellung des zweistufigen Prozesses [5]

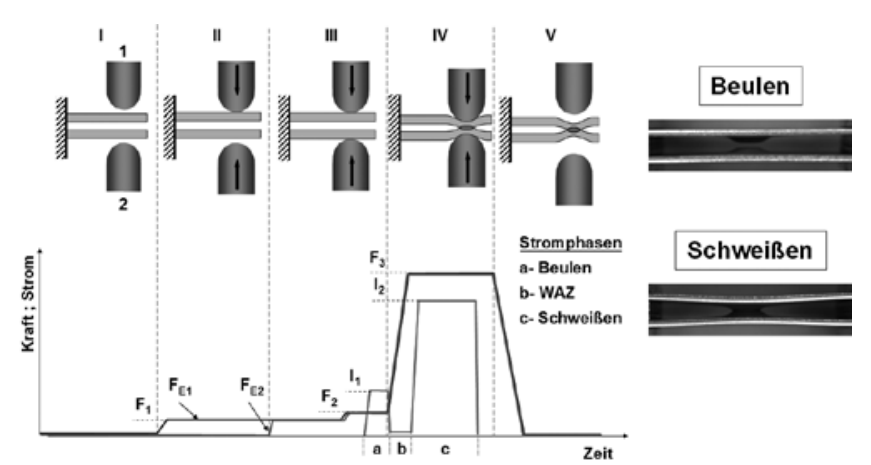

Abb. 11. Zeit-Strom-Kraft-Diagramm des zweistufigen Widerstandspunktschweißprozess [5]
Prozess beginnt mit einer synchronen Bewegung beider Elektroden in der Richtung der Bauteile. Hierbei wird nur eine sehr geringe Elektrodenkraft aufgebaut. Im Falle eines Spaltes wird die Elektrodenkraft leicht erhöht und anschließend der Strom zum Warmbeulen eingeschaltet. Der Schweißflansch bleibt weiterhin symmetrisch belastet. Der Strom fließt bis zum Kontakt beider Bleche. Während der Wärmeausgleichzeit wird die Fügestelle über die kontaktierende Elektrodenkappen gekühlt und die Elektrodenkraft auf den Wert zur eigentlichen Schweißlinsenbildung gesteigert. Eine endgültige Verbindung der Bleche geschieht während des Schweißens der vorgeformten Bauteile. Die herkömmliche Nachhaltezeit schließt den zweistufigen Prozess ab.

Adaptive Qualitätsregelsysteme zum Widerstandspunktschweißen ermöglichen eine individuelle Anpassung elektrischer Schweißparameter während der Schweißlinsenbildung für jeden einzelnen Schweißpunkt. Die Integration des zweistufigen Schweißprozesses auf derselben Plattform garantiert zusätzlich mit Strom- und Kraftprogrammen eine prozesssichere Spaltüberbrückung unter Berücksichtigung von Randbedingungen der Automobilindustrie.

\section{Versuchsaufbau und -durchführung}

Zur Analyse des neuartigen Regelkonzeptes wurde in der Konzernforschung der Volkswagen $A G$ eine seriennahe Schweißanlage mit einer C-Roboterschweißzange um eine zusätzliche Schnittstelle für einen übergeordneten Regler, verschiedene Sensoren zu Erfassung von Prozessparameter und einen Zangenausgleich mit einem Servomotor erweitert. Die Abbildung 12 zeigt schematisch den Aufbau der Versuchsanlage.

Die veränderte Prozessführung wurde vom übergeordneten Steuerrechner auf PC-Basis mittels eines speziellen LabView-Programms koordiniert. Auf der Abbildung 13 sind Verläufe von Stell-, Messwerte der Elektrodenkräfte und der Stromstärkesollwerte während des zweistufigen Schweißvorganges mit der Umformung und anschließender Schweißlinsenbildung am Beispiel des HX340LAD +Z100 in 1,0 mm Materialstärke abgebildet. Der Prozessverlauf entspricht dem aus der Abbildung 11.

Im Rahmen dieser Untersuchung erfolgte keine Taktzeitoptimierung der Versuchsanlage. Anhand der praktischen Erfahrung wurde die maximal zulässige Warmumformung auf 1000 ms begrenzt. Die Wärmeausgleichzeit und die Nachhaltezeit lagen werkstoffunabhängig entsprechend bei 500 und $300 \mathrm{~ms}$.

Für die Schweißversuche mit der beidseitigen Flanschspannung wurden Blechproben von 200 x 45 $\mathrm{mm}$ hergestellt und in einem Spezialwerkzeug eingespannt, wie in der Abbildung 14 schematisch dargestellt. Der Passungsabstand P blieb während aller 


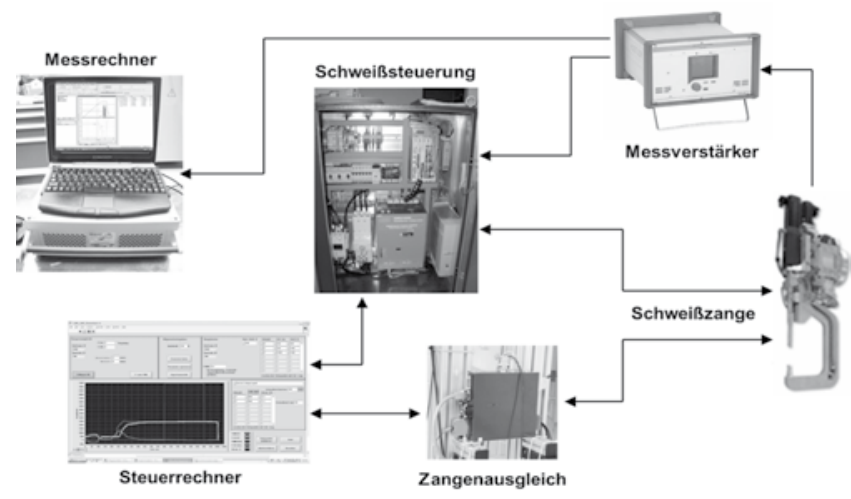

Abb. 12. Schematischer Aufbau der Versuchsanlage

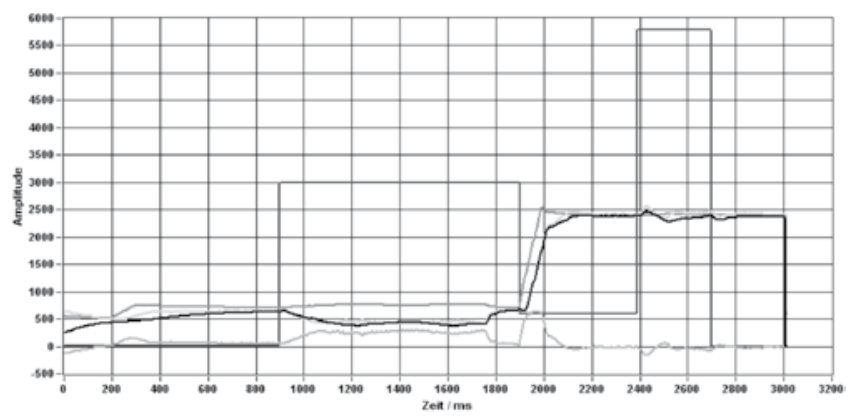

Abb. 13. Prozessgrößenverlauf aus der LabView-Bedienoberfläche beim zweistufigen Schweißvorgang am Beispiel HX340LAD+Z100 in $1,0 \mathrm{~mm}$ Materialstärke

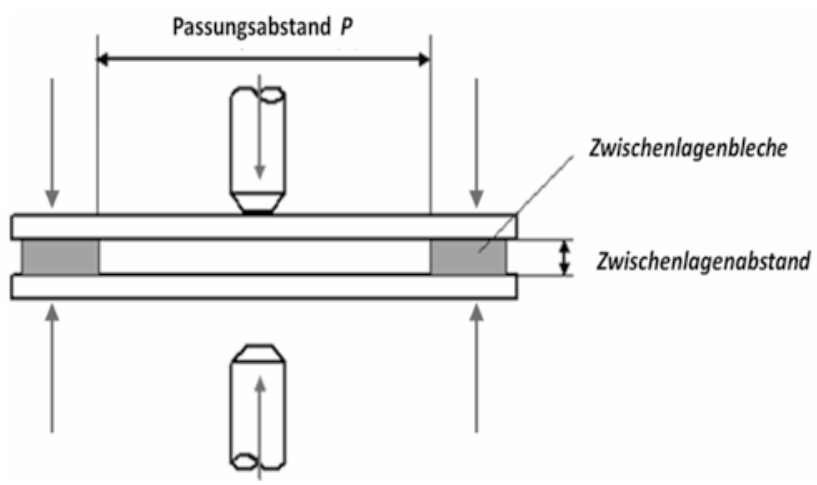

Abb. 14. Einspannung der Versuchsbleche

Versuche konstant und betrug $50 \mathrm{~mm}$. Der Spalt zwischen den zu fügenden Blechen wurde ebenfalls auf $5 \mathrm{~mm}$ festgelegt. Durch eine sehr hohe Steifigkeit der Einspannvorrichtung und eine beidseitige Blecheinspannung konnte ein praxisnahes Verhalten des Schweißflansches an größeren Baugruppen abgebildet werden. Anhand der Werkstoffanalyse der Fahrzeugkarosserie wurden für die Untersuchung repräsentativ Stähle HX340LAD+Z100 in $1,0 \mathrm{~mm}$ und 22MnB5+AS150 in 1,5 mm Materialstärke ausgewählt.

\section{Warmbeulen}

Bei der Analyse der Beulphase wurde die Möglichkeit zur Spaltüberbrückung mittels einer überwiegend lokalen Verformung des Schweißflansches um die
Fügestelle geprüft. Ein optimaler Prozess sollte bereits vor der tatsächlichen Schweißlinsenbildung eine prozesssichere Bauteilkontaktierung gewährleisten. Dabei wurden die Elektrodenkraft und die Stromstärke variiert.

Die Abbildung 15 stellt die Verteilung der lokalen und globalen Flanschverformung im entlasteten $\mathrm{Zu}$ stand nach dem Warmbeulen für ausgewählte Prozessparameter dar. Trotz einer geringen Flanschsteifigkeit des HX340LAD+Z100 konnte der Spalt innerhalb der vorausgesetzten Prozesszeit erst ab der Elektrodenkraft von $0,7 \mathrm{kN}$ überbrückt werden. Im Allgemeinen kam es mit der zunehmenden Elektrodenkraft zu einer größeren Globalverformung des Flansches. Bei einer geringeren Anpresskraft trat ein erheblicher Anstieg des elektrischen Widerstandes an der Kontaktstelle der Bleche mit den Elektroden und in der Fügezone auf. Demzufolge nahm mit der eingebrachten Energie der Elektrodenkappenverschleiß und die Verschmutzung der Blechoberfläche wesentlich zu. Außerdem wurden vermehrt Schweißspritzer in der Stromphase zu beobachtet.

Beim höchstfesten 22MnB5+AS150 konnte mit dem herkömmlichen Schweißvorgang wegen einer sehr hohen Streckgrenze und Flanschsteifigkeit keine Schweißlinse gebildet werden. Alle Schweißversuche mit 1 und 1,5 kN Elektrodenkraft führten auf Grund eines höheren Werkstoffwiderstandes und einer geringeren Wärmeleitfähigkeit zum Schmelzen des Versuchsmaterials und der Elektrodenkappe. Die Schweißspritzer waren nicht zu verhindern. Nach einer Elektrodenkrafterhöhung auf $2 \mathrm{kN}$ konnte der eingestellte Spalt sowohl mit der Stromstärke von $4 \mathrm{kA}$ als auch 4,5 kA ohne Materialverluste überwindet werden. Erst ab

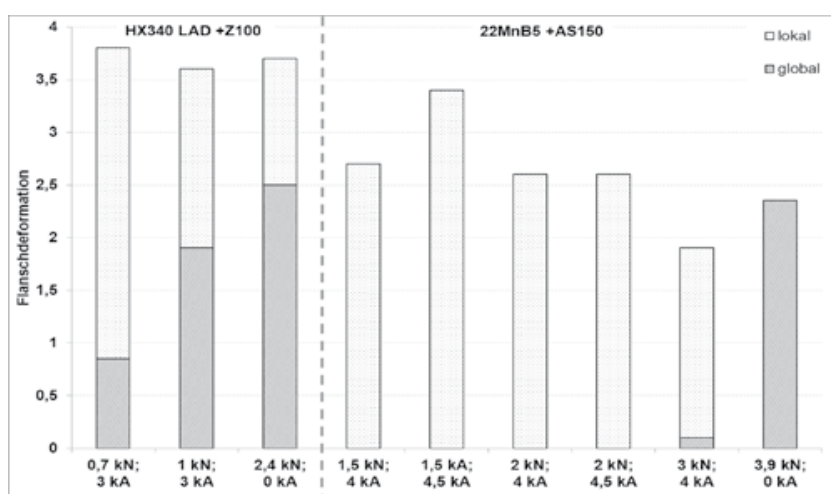

Abb. 15. Verformungsanteile bei Variation der Elektrodenkraft und Stromstärke während des Beulens

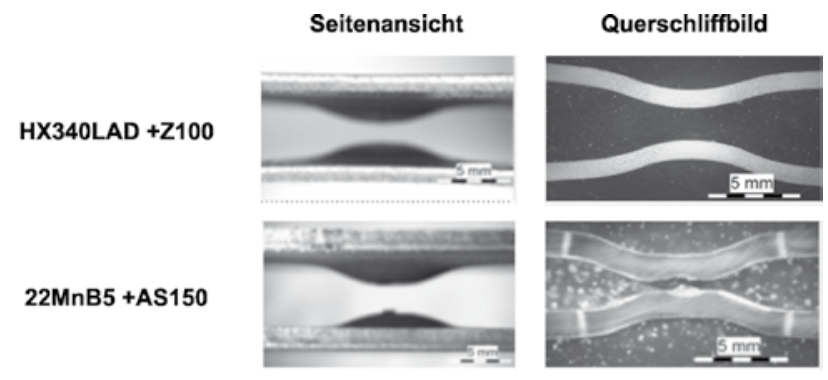

Abb. 16. Flanschgeometrie nach dem Warmbeulen mit optimierten Schweißparametern 
3 kN Elektrodenkraft wurde der globale Anteil der Flanschverformung festgestellt.

Für den hochfesten Stahl wurde bereits mit der Elektrodenkraft von $0,7 \mathrm{kN}$ und Stromstärke von 3,0 kA der eingestellte Spalt überbrückt und dabei die geringste globale und plastische Deformation des Flansches gemessen. Da die Verformungskomponenten beim höchstfesten, warm umgeformten Stahl weitgehend unabhängig von der Stromstärke waren, entschied die Sichtprüfung äußerer Befunde über die Auswahl den Parametersatz der Elektrodenkraft von 2,0 kN und Stromstärke von $4 \mathrm{kA}$ zur Untersuchung der Schweißlinsenbildung des zweistufigen Schweißprozesses. Die Seitenansicht des Schweißflansches sowie das metallografische Schliffbild beider Werkstoffe nach dem Warmbeulen sind im unbelasteten Zustand in der Abbildung 16 für ausgewählte Prozessparameter präsentiert.

\section{Schweißlinsenbildung}

Die Bewertung der Schweißpunktqualität und der Schweißflanschgeometrie wurde für den optimierten Parametersatz aus der Analyse der Warmbeulung durchgeführt. Als Ausgangswerte für den Schweißvorgang des zweistufigen Schweißprozesses wurden berechnete Schweißparameter für den herkömmlichen Schweißprozess verwendet.

Mit dem zweistufigen Schweißvorgang konnte mit optimierten Beulparametern am HX340LAD+Z100 die lokale Flanschverformung von ca. $50 \%$ der Gesamtdeformation erreicht werden. Bei den restlichen Parametersätzen lag dieser Anteil unter 30\%. Die Abbildung 17 zeigt die Flanschoberfläche und die Seitenansicht um die Fügestelle des zweistufigen Prozesses nach der Optimierung der Prozessparameter beider Prozessphasen im Vergleich zum herkömmlichen Schweißvorgang mit einer manuellen Parameteranpassung.

Der größte Unterschied nach der Sichtprüfung lag in der Größe der Wärmeeinflusszone. Diese ist beim herkömmlichen Prozess deutlich geringer, da mit dem langen Beulvorgang der Fügestelle eine zusätzliche Energie zugeführt wird. Erwartungsgemäß trat beim Schweißen ohne das Warmbeulen eine größere Globalverformung auf. Die zweite Prozessphase erhöhte den lokalen Verformungsanteil zusätzlich um ca. $20 \%$. Das Bauteilkontaktieren erfolgte jedoch überwiegend durch eine globale Deformation.

Wegen einer größeren Kontaktfläche des Werkstücks mit den Elektrodenkappen und in der Fügeebene entstand nach dem Warmbeulen und der anschließenden Schweißung mit berechneten Schweißparametern ebenso keine normgerechte Schweißlinse, siehe Abbildung 18. Demzufolge ist eine Anpassung der Schweißparameter für die veränderte Geometrie des Schweißflansches beim zweistufigen Schweißprozess notwendig. Mit dem gleichen Parametersatz wurde der mittlere Punktdurchmesser nach dem gängigen Schweißprozess von etwa $2,5 \mathrm{~mm}$ erreicht, weil die
Schweißlinsenbildung nach der unkontrollierten Spaltüberbrückung erst begann.

Der Vergleich beider Prozessführungen zeigte unterschiedliche Deformationen im Bereich des Schweißpunktes. Aus Schliffbildern in der Abbildung 18 war die überwiegend lokale Deformation aus dem Warmbeulen auch der der Schweißung deutlich zu erkennen.

Wie bereits erwähnt, konnte mit dem herkömmlichen Schweißprozess wegen eines enormen Materialverlustes keine Schweißlinse gebildet werden, Abbildung 19. Obwohl es auf der Blechinnenseite zum Schmelzen und Fügen beider Versuchsbleche kam, entstand jedoch aufgrund der im Gefüge eingefrorenen Spannungen keine dauerhafte Schweißverbindung.

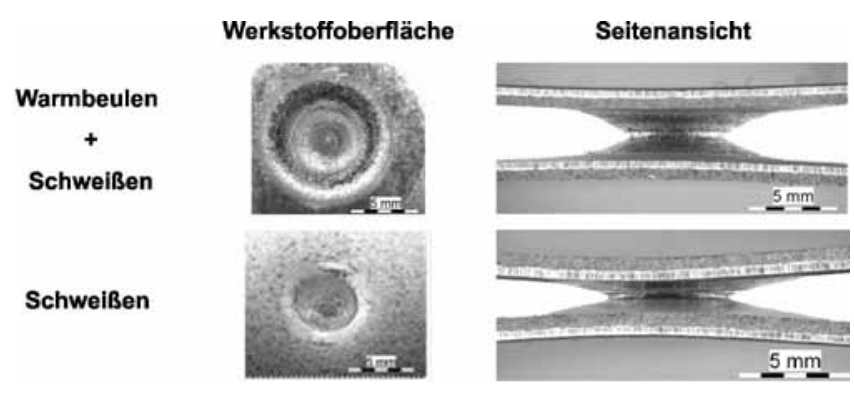

Abb. 17. Oberflächenzustand und die Flanschgeometrie nach dem herkömmlichen und zweistufigen Schweißvorgang am HX340LAD +Z100 in 1,0 mm Materialstärke

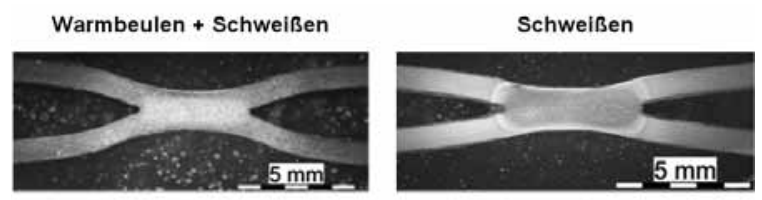

Abb. 18. Vergleich der Schweißlinsenbildung mit berechneten Schweißparameter des zweistufigen (links) und herkömmlichen (rechts) Prozesses für HX340LAD +Z100
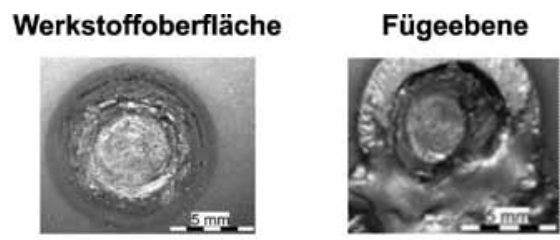

Abb. 19. Oberflächenzustand des Schweißflansches (links) und die Fügeebene (rechts) nach dem herkömmlichen Schweißvorgang am $22 \mathrm{MnB} 5+\mathrm{AS} 150$ in 1,5 mm Materialstärke
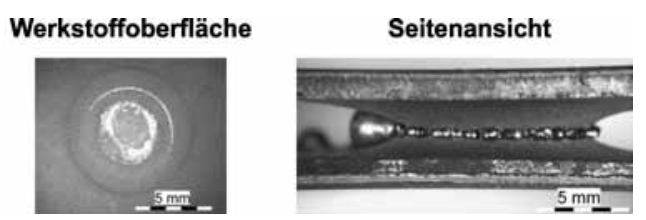

Abb. 20. Oberflächenzustand und die Flanschgeometrie nach dem zweistufigen Schweißvorgang am 22MnB5 +AS150 in 1,5 mm Materialstärke
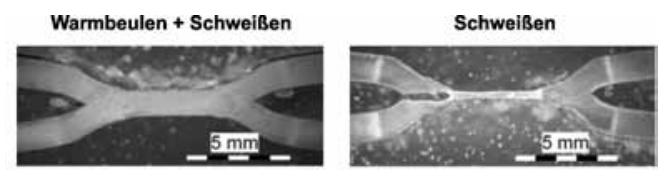

Abb. 21. Vergleich der Schweißpunktgeometrie mit berechneten Schweißparametern des zweistufigen (links) und herkömmlichen (rechts) Prozesses für 22MnB5 +AS150 
Während des Warmbeulens kam es zur Verringerung des Spaltes auf 2,4 mm, was eine Bauteilkontaktierung mit der Schweißkraft bei einer höheren Stromstärke in der nächsten Prozessphase ermöglichte. Die Messung der Blechdeformation nach dem Gesamtprozess ergab den Elektrodenweg von insgesamt $5,6 \mathrm{~mm}$, wobei der lokale Anteil bei $70 \%$ lag. Trotz des zweistufigen Schweißvorganges entstand während der Schweißlinsenbildung eine geringe Werkstoffauspressung, wie in der Abbildung 20 dargestellt. Außerdem kam es zur Schweißspritzenbildung in der Fügezone.

Ähnlich dem hochfesten Werkstoff war eine geringe Anpassung von Schweißparametern notwendig, um eine normgerechte Schweißlinse mit dem zweistufigen Prozess zu erreichen. Wegen einer
Vergrößerung Kontaktfläche führte eine Verlängerung der Prozesszeit nicht zur Verbesserung. Erst nach der Erhöhung der Stromstärke von 9 auf $11 \mathrm{kA}$ entstand der Schweißpunkt mit dem Durchmesser von 6,3 mm. Dabei blieb die Schweißzeit und die Elektrodenkraft unverändert bei entsprechend $400 \mathrm{~ms}$ und $3,9 \mathrm{kN}$. Die Stromerhöhung verursachte eine stärkere Erwärmung des Werkstoffes und dadurch ein tieferes Einsinken der Elektroden. Trotz einer nur geringen Einschnürung der Wanddicke im Randbereich der Schweißlinse wurde die gesamte Blechdicke am Schweißpunkt um fast $50 \%$ reduziert. Die verbliebene Restblechdicke im Bereich des Elektrodeneindrucks ist im Vergleich zum herkömmlichen Schweißvorgang in der Abbildung 21 gezeigt.

\section{Zusammenfassung}

Die Untersuchungen am toleranzbehafteten Schweißflansch haben beim herkömmlichen Schweißvorgang sogar beim Tiefziehstahl große Probleme mit der Schweißpunktqualität in Bezug auf die $\mathrm{Fe}$ stigkeit und das Aussehen verdeutlicht. Die unkontrollierte Flanschdeformation bewirkt nicht nur eine Verkürzung der Prozesszeit zur Schweißlinsenbildung, sondern verändert auch die Kontaktbedingungen in der Fügestelle. Diese Problematik nimmt mit der Werkstofffestigkeit und Steifigkeit zu, weil kein Blechkontakt mit der zur Verfügung stehenden Elektrodenkraft hergestellt wird. In diesem Fall verläuft der Prozess sehr unstabil.

Der untersuchte Lösungsansatz trennt den Schweißvorgang abhängig von der Spaltsituation in das Warmbeulen und das Schweißen. Im ersten Schritt wird der Schweißflansch lokal verformt und erst in der zweiten Phase eine normgerechte
Schweißlinse gebildet. Durch eine werkstoffspezifische Anpassung der Schweißparameter wurde eine Verringerung der globalen Verformung der Fügestelle erreicht. Der so verformte Schweißflansch wurde anschließend quasi spannungsfrei in der zweiten Prozessphase verschweißt.

Zusammenfassend kann man feststellen, dass beim zweistufigen Schweißprozess eine symmetrische, poren- und rissfreie Schweißlinse entsteht. Ebenso erschienen keine Poren oder Rissen in der Wärmeeinflusszone oder im Grundwerkstoff. Durch die zusätzliche Prozessphase wird mehr Energie zugeführt, wodurch sich der Elektrodeneindruck im Werkstück vergrößert. Hierbei kommt es beim Spalt zu einer genauen Anpassung der äußeren Blechoberfläche an die Elektrodenkappenform. Der zweistufige Prozess hat sich als besonders vorteilhaft beim Fügen von höchstfesten Werkstoffen erwiesen.

\section{Literatur}

[1] Jüttner S., Meyer R.: Leichtbau - Herausforderungen für die Fügetechnik. Volkswagen AG, Aktionstag Innovation im Automobilbau, Dresden, 2007.

[2] Müller S.: Fügetechnologien im Karosseriebau - Status und Trends. Konferenz: Automobilbau - Trends in der Füge- und Werkstofftechnik, 2008.

[3] Bergmann J.P.: Łączenie stali ocynkowanej: stan aktualny i perspektywy. Przegląd Spawalnictwa, 8-9/2004, s. 76.

[4] Füssel U., Jüttner S., Meyer R.: Wirkung von Toleranzen im Karosseriebau auf die Fügetechnik. Zwischensitzung IIW SC Automotive and Road Transport; Berlin am 19-20.03.2008

[5] Baryliszyn P., Noack Th., Füssel U., u.a.: Erweiterung der Schweißzangenregelung auf Basis der Elektrodenreferenzkraft. 21. DVS-Sondertagung, Widerstandspunktschweißen 2010, Duisburg, 2010.

[6] Baryliszyn P., Jüttner S., Füssel U.: Neuartiges Regelsystem zum Widerstandspunktschweißen von Karosseriebauteilen mit Maßabweichungen. Große Schweißtechnische Tagung, 15. September 2009.

[7] TL4225: Legierter Vergütungsstahl 22MnB5, ungeschichtet oder vorbeschichtet; Werkstoffanforderungen an Halbzeuge und Bauteile. Stand 2006.

[8] PN-EN 10025: Wyroby walcowane na gorąco ze stali konstrukcyjnych. Stand 2007.

[9] Woestmann H.: Moderne Stahlfeinbleche für den Automobilleichtbau. Aktueller Stand und Ausblick in die Zukunft. Erfahrungsaustauschgruppe PZS-Werkzeuge, Lüdenscheid, 2005.

[10] Overrath J.: Schweißen von warmumgeformten Bauteilen. 11. ifs - Kolloquium, Braunschweig, 2007.

[11] PV 6702: Punktschweißverbindungen. Festigkeitsprüfung Stahlwerkstoffe. Stand 2004.

[12] DVS-RichtlinieDVS 2902-3: Widerstandspunktschweißen von Stählen bis $3 \mathrm{~mm}$ Einzelblechdicke. Konstruktion und Berechnung. Stand 1991.

[13] Piwowar S.: Spawanie i zgrzewanie elektryczne. WSiP, Warszawa, 1966.

[14] ISO 6520-2: Welding and allied processes: Classification of geometric imperfections in metallic materials. Part 2: Welding with pressure. Stand 2001.

[15] VW 01105-1: Widerstandspunktschweißen. Konstruktion, Berechnung. Stand 2007. 